\title{
Espasmo hemifacial: experiencia en un centro terciario
}

\section{Hemifacial spasm: our experience in a tertiary referral hospital}

\author{
Eduard D. Neumann R. ${ }^{1}$, Alfons Gutiérrez B. ${ }^{1}$, Julia De Juan B. ${ }^{1}$, \\ Albert Pujol O. ${ }^{1}$, María del Prado Venegas P. ${ }^{1}$ César Orús D. ${ }^{1}$
}

\section{Resumen}

El espasmo hemifacial consiste en un infrecuente trastorno del movimiento que afecta a la musculatura inervada por el nervio facial, en especial la de la hemicara superior. Existen dos formas clínicas, una primaria en la que la causa subyacente está representada por un conflicto neurovascular, y una secundaria, en la que la alteración del nervio facial es producida por algún tipo de lesión ocupante de espacio. Resulta de especial interés para el otorrinolaringólogo conocer esta entidad y ser capaz de diferenciar ambas formas clínicas dado el diferente enfoque terapéutico que pueden requerir. Aportamos una visión general de esta patología repasando su epidemiología y fisiopatología, además, de nuestra experiencia en forma de una serie de cuatro casos que ilustran las variadas formas de presentación de esta entidad, así como los signos y síntomas de alarma que pueden ayudar a realizar un correcto diagnóstico y manejo.

Palabras clave: espasmo hemifacial, trastorno movimiento, conflicto neurovascular, nervio facial.

\begin{abstract}
Hemifacial spasm consists of an unfrecuent movement disorder involving facial muscles, especially those from the upper half of the face. Two different clinical presentations are described. Primary hemifacial spasm is defined by a neurovascular conflict, and secondary occurs when facial nerve is damaged by a space occupying lesion. It is of special interest for the otorhinolaryngologist to get to know about this condition and to be able to acknowledge its clinical presentations due to the different therapeutical approach that may be needed for each of them. We contribute with a general vision of this entity reviewing its epidemiology and patophysiology. Furthermore, we show our experience by sharing a four-case series which we believe to illustrate the different ways of presentation as well as the alarm signs and symptoms that may be helpful in order to accomplish an accurate diagnose and treatment.
\end{abstract}

Keywords: hemifacial spasm, movement disorder, neurovascular conflict, facial nerve.

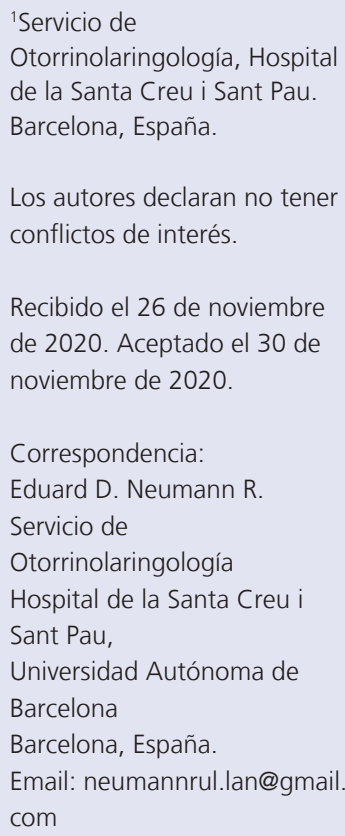

Los autores declaran no tener conflictos de interés.

Recibido el 26 de noviembre de 2020. Aceptado el 30 de noviembre de 2020 .

Correspondencia:

Eduard D. Neumann R.

Servicio de

Otorrinolaringología

Hospital de la Santa Creu i

Sant Pau,

Universidad Autónoma de

Barcelona

Barcelona, España.

Email: neumannrul.lan@gmail. com

\section{Introducción}

El espasmo hemifacial consiste en un trastorno del movimiento de la musculatura facial caracterizado por contracciones involuntarias de los músculos inervados por el nervio facial. Las contracciones pueden ser breves y localizadas o bien ocurrir en salvas e incluso ser persistentes provocando una contractura de toda la hemicara de forma tónica. La musculatura que con más frecuencia se ve afectada es la orbicular de los ojos y el área cigomática ${ }^{1}$.
El primer caso descrito de esta entidad sea posiblemente el de una mujer de 56 años descrito por el Dr. Schultze en 1875. El estudio post mortem de la paciente reveló un aneurisma gigante de la arteria vertebral que comprimía el nervio facial. Pocos años después, el Dr. Gowers en 1899 también describió un caso similar ${ }^{2}$. Aunque su prevalencia es difícil de estimar dado el infradiagnóstico, la literatura está de acuerdo en que la prevalencia es aproximadamente de 9,8/100.000 habitantes. La edad media de inicio del cuadro suele estar entorno 
a la $4^{\mathrm{a}}-6^{\mathrm{a}}$ década de la vida según la mayoría de las series. Existe además una diferencia de incidencia en cuanto a sexos, estando definida una predominancia del sexo femenino con una ratio de hasta 3:1 según la serie ${ }^{3,4}$. Casos familiares han sido descritos, aunque representan como mucho un 2\%-3\% en las series más amplias ${ }^{1}$.

Existen dos formas clínicas de espasmo hemifacial: una primaria y una secundaria. La primaria es el resultado de una compresión vascular sobre el nervio facial. Las formas secundarias agrupan un conjunto de entidades en las que ocurre una alteración del propio nervio facial a lo largo de su recorrido desde el conducto auditivo interno hasta el foramen estilomastoideo ${ }^{5}$. Entre ellas, lesiones del ángulo pontocerebeloso o malformaciones arteriovenosas que afecten al tronco cerebral $^{6}$. También infecciones como una otitis media descontrolada o un colesteatoma con afectación de fosa craneal posterior pueden corresponder a la causa subyacente en las formas secundarias.

\section{Fisiopatología}

El nervio facial surge del tronco del encéfalo en una zona situada entre la protuberancia y el bulbo raquídeo. La porción de nervio que emerge del tronco recibe el nombre de root exite zone (REZ). Al final de dicha zona de emergencia, existe lo que se conoce como transition zone (TZ), donde se produce la transición de oligodendrocitos (mielina central) a células de Schwann (mielina periférica). Esta zona es especialmente vulnerable a la compresión dado que no presenta una cobertura de epineuro ni perineuro ${ }^{2}$.

En las formas primarias, el conflicto neurovascular suele deberse a un loop arterial. Las series clásicas sugieren que la arteria protagonista en la mayoría de los casos es la arteria cerebelosa antero inferior (AICA), seguida de la arteria cerebelosa postero inferior (PICA) y de la arteria vertebral. Sin embargo, en una revisión del año $2017^{7}$ que incluye 11 series $(\mathrm{n}=2.829$ casos), el vaso que más frecuentemente se vio implicado fue la PICA $(47,2 \%)$ seguido de la AICA $(45,9 \%)$ y la arteria vertebral $(17,5 \%)$. No obstante, los porcentajes ofrecidos por los diferentes autores son, en ocasiones, dispares. El trabajo revisado argumenta que este hecho puede deberse a una dificultosa identificación intraoperatoria del vaso involucrado ${ }^{7}$.

La forma en que esta hiperexcitabilidad se propaga está en debate. Existen dos teorías defendidas. La teoría periférica sugiere que la compresión vascular provoca una desmielinización responsable de descargas nerviosas ectópicas y espontáneas. Esta teoría propone, además que dichos impulsos se transmiten de forma lateral entre las diferentes ramas del nervio facial, a través de lo que se conoce en la literatura como cross-talk que precisamente sería más factible en la TZ, donde recordemos, las fibras nerviosas no disponen de tejido conectivo y son más susceptibles a la compresión. A nivel electromiográfico esta respuesta puede determinarse y se conoce como lateral spread response $(\mathrm{LSR})^{2}$. Al estimular la rama temporal, se obtiene una respuesta directa en el músculo frontal y una respuesta anormal (LSR) en el músculo inervado por la rama mandibular. De esta manera se pone de manifiesto este fenómeno de comunicación nerviosa ${ }^{1}$. La teoría central propone que la hiperexcitabilidad viaja de forma retrógrada hasta el núcleo motor del nervio facial a través del cual, las LSR, se transmiten de forma retrógrada hacia otras neuronas de otras ramas del nervio facial ${ }^{1}$.

\section{Diagnóstico y formas secundarias en ORL}

Las contracciones involuntarias de la hemicara superior son fácilmente identificables y el "otro signo de Babinski” es un hallazgo que ha demostrado tener gran sensibilidad (86\%) y especificidad (100\%) en el diagnóstico del espasmo hemifacial. Consiste en la contracción simultánea e involuntaria de la musculatura orbicular de los ojos y la musculatura frontal provocando un cierre del ojo a la vez que se eleva la ceja del paciente ${ }^{4}$.

Herramientas como la EMG y la resonancia magnética (RM) son esenciales para confirmar el diagnóstico y diferenciar las formas primarias de las secundarias. La RM de alta resolución con secuencias en T2 y T1 con contraste son imperativas hoy en día, especialmente en el contexto de la planificación quirúrgica ${ }^{8}$. 
La historia clínica, junto con una exploración física y las pruebas complementarias adecuadas, facilitarán el proceso del diagnóstico diferencial del espasmo hemifacial. Entre ellos, cabe mencionar el blefarospasmo, el espasmo posparálisis y las mioquimias. Otras entidades como los espasmos psicógenos, los tics faciales o las distonías oromandibulares pueden dificultar el proceso diagnóstico por ser entidades imitadoras ${ }^{1,4}$.

Como otorrinolaringólogos nos interesa identificar aquellos casos que puedan tratarse de un espasmo hemifacial secundario. Deben llamar la atención datos clínicos de la anamnesis como la hipoacusia, los acúfenos o la otorrea que acompañen a un espasmo hemifacial. En el mismo sentido, un espasmo hemifacial que progresa a parálisis fácil debe alertarnos. En estos casos, es obligada la realización de una RM para descartar la presencia de lesiones que afecten al nervio facial como, por ejemplo, una lesión que ocupe el ángulo ponto cerebeloso.

\section{Manejo: médico y quirúrgico}

El objetivo del manejo médico es el de reducir el número y la magnitud de las contracciones musculares anómalas. El enfoque que se plantea de forma inicial se basa en la utilización de la toxina botulínica, que evita la conducción de impulsos neuromusculares provocando así una parálisis muscular transitoria?.

Según la literatura se trata de un tratamiento efectivo y con pocos efectos secundarios. A saber, se han descrito ptosis palpebral (15\%), paresia facial transitoria o leves asimetrías $(23 \%)^{9}$. El tipo de toxina más utilizada es la toxina tipo A y se suele inyectar entre 10-34 unidades en la musculatura orbicular de los ojos, en el corrugado frontal, en la musculatura cigomática, en buccinador y masetero. El contrapunto principal de este tratamiento es su efecto temporal ${ }^{10}$.

En un número importante de trabajos entre los que destaca un estudio multicéntrico de 10 años de duración, se reportan tasas de éxito que van desde el $75 \%$ hasta el $100 \%{ }^{9,11}$.

El enfoque quirúrgico se puede plantear en pacientes en los que la toxina botulínica no ha sido efectiva o que directamente rechazan esa opción, así como en los casos severos. La microdescompresión vascular (MVD, por sus siglas en inglés) persigue evitar el con- tacto entre el vaso y el nervio facial. Algunos trabajos ${ }^{2}$ defienden que las LSR desaparecen de forma intraoperatoria al realizarse esta intervención.

La tasa de éxito según series grandes es de entre el 85\%-90\%. No obstante, es importante tener en cuenta los potenciales riesgos de este tipo de intervención que, recordemos, se realiza de forma electiva en una entidad benigna. Entre ellos cabe resaltar el propio riesgo anestésico, la parálisis facial permanente (1\%-2\%), la hipoacusia permanente (2\%-3\%) y la fístula de líquido cefalorraquídeo $(<2 \%)^{12}$.

\section{Casos Clínicos}

En nuestro servicio contamos con cuatro casos de espasmo hemifacial de los cuales tan solo el primero corresponde a un espasmo hemifacial primario. Los datos relevantes de cada caso se encuentran resumidos en la tabla adjunta (Tabla 1). Resulta especialmente relevante el progreso clínico de los espasmos hemifaciales hacia parálisis facial observado en el 50\% de los casos. En el proceso diagnóstico fue crucial el papel de la RM en todos los casos.

\section{Conclusión}

El espasmo hemifacial es una entidad poco frecuente, pero pensamos que, desde el punto de vista otorrinolaringológico, más allá del debate en torno a la fisiopatología, debemos conocer su existencia y saber diferenciar sus formas clínicas primarias y especialmente las secundarias. Las formas clínicas secundarias deben ser sospechadas si existe clínica acompañante. El estudio mediante RM es de obligada realización en estos casos. Los casos expuestos pretenden hacer visible las diferentes formas de presentación que puede tener esta enfermedad, así como los signos de alarma que nos deben alertar. Por otro lado, pretenden mostrar que la indicación quirúrgica debe adecuarse siempre a la magnitud de los síntomas dadas las potenciales secuelas. Además, remarcar que el tratamiento médico basado en toxina botulínica tipo A puede ser una opción satisfactoria a pesar de no representar una alternativa curativa. 
Tabla 1. Relación detallada de los casos descritos. EH espasmo hemifacial, MVD descompresión microvascular

\begin{tabular}{|c|c|c|c|c|c|c|c|}
\hline Número & $\begin{array}{l}\text { Gér } \\
\text { y e }\end{array}$ & $\begin{array}{l}\text { ero } \\
\text { dad }\end{array}$ & Forma clínica & Síntoma inicial & Etiología & Tratamiento & Estado actual \\
\hline 1 & $\mathrm{H}$ & 62 & $\begin{array}{c}\mathrm{EH} \\
\text { primario }\end{array}$ & $\begin{array}{c}\text { EH } \\
\text { superior }\end{array}$ & AICA & $\begin{array}{l}\text { Toxina botulínica } \\
\text { Cirugía MVD }\end{array}$ & Sin síntomas \\
\hline 2 & $\mathrm{H}$ & 45 & $\begin{array}{c}\text { EH } \\
\text { secundario }\end{array}$ & $\begin{array}{c}\text { EH progresivos a } \\
\text { parálisis }\end{array}$ & $\begin{array}{l}\text { Recidiva } \\
\text { colesteatoma } \\
\text { congénito }\end{array}$ & $\begin{array}{c}\text { Petrosectomía } \\
\text { neurorrafia XII-VII }\end{array}$ & Asimetría facial \\
\hline 3 & $\mathrm{H}$ & 40 & $\begin{array}{c}\text { EH } \\
\text { secundario }\end{array}$ & $\begin{array}{c}\text { Hipoacusia } \\
\text { EH progresivos } \\
\text { a parálisis }\end{array}$ & $\begin{array}{l}\text { Neurinoma } \\
\text { Intra } \\
\text { canalicular }\end{array}$ & $\begin{array}{c}\text { Exéresis } \\
\text { translaberíntica } \\
\text { neurorrafia XII-VII }\end{array}$ & $\begin{array}{l}\text { Parálisis } \\
\text { facial House- } \\
\text { Brackmann III }\end{array}$ \\
\hline 4 & M & 62 & $\begin{array}{c}\text { EH } \\
\text { secundario }\end{array}$ & $\begin{array}{c}\text { Hipoacusia } \\
\text { acufenos } \\
\text { inestabilidad } \\
\text { EH }\end{array}$ & $\begin{array}{l}\text { Neurinoma } \\
\text { intra } \\
\text { canalicular }\end{array}$ & $\begin{array}{l}\text { Toxina botulínica } \\
\text { periódica }\end{array}$ & $\begin{array}{l}\text { Control de } \\
\text { síntomas }\end{array}$ \\
\hline
\end{tabular}

\section{Bibliografía}

1. Lefaucheur JP, Ben Daamer N, Sangla S, Le Guerinel C. Diagnosis of primary hemifacial spasm. Neurochirurgie. 2018;64(2):82-86. doi: 10.1016/j.neuchi.2017.12.003.

2. Lefaucheur JP. New insights into the pathophysiology of primary hemifacial spasm. Neurochirurgie. 2018;64(2):87-93. doi: 10.1016/j.neuchi.2017.12.004.

3. Martinez AR, Nunes MB, Immich ND, et al. Misdiagnosis of hemifacial spasm is a frequent event in the primary care setting. Arq Neuropsiquiatr. 2014;72(2):119-122. doi: 10.1590/0004$282 X 20130227$.

4. Lu AY, Yeung JT, Gerrard JL, Michaelides EM, Sekula RF Jr, Bulsara KR. Hemifacial spasm and neurovascular compression. Scientific World Journal. 2014;2014:349319. doi: 10.1155/2014/349319.

5. Colosimo C, Bologna M, Lamberti S, et al. A comparative study of primary and secondary hemifacial spasm [published correction appears in Arch Neurol. 2006 Sep;63(9):1241. Avanzino, Lucio [corrected to Avanzino, Laura]; Marinelli, Laura [corrected to Marinelli, Lucio]]. Arch Neurol. 2006;63(3):441-444. doi: 10.1001/archneur.63.3.441.

6. Han IB, Chang JH, Chang JW, Huh R, Chung SS.
Unusual causes and presentations of hemifacial spasm. Neurosurgery. 2009;65(1):130-137. doi: 10.1227/01.NEU.0000348548.62440.42.

7. Mercier P, Sindou M. The conflicting vessels in hemifacial spasm: Literature review and anatomicalsurgical implications. Neurochirurgie. 2018;64(2):94100. doi: 10.1016/j.neuchi.2018.01.004.

8. Hermier M. Imaging of hemifacial spasm. Neurochirurgie. 2018;64(2):117-123. doi: 10.1016/j.neuchi.2018.01.005.

9. Chaudhry N, Srivastava A, Joshi L. Hemifacial spasm: The past, present and future. J Neurol Sci. 2015;356(12):27-31. doi: 10.1016/j.jns.2015.06.032.

10. Safarpour Y, Jabbari B. Botulinum Toxin Treatment of Movement Disorders. Curr Treat Options Neurol. 2018;20(2):4. Published 2018 Feb 24. doi: 10.1007/ s11940-018-0488-3.

11. Defazio G, Abbruzzese G, Girlanda P, et al. Botulinum toxin A treatment for primary hemifacial spasm: a 10-year multicenter study. Arch Neurol. 2002;59(3):418-420. doi: 10.1001/archneur.59.3.418.

12. Sindou M, Mercier P. Microvascular decompression for hemifacial spasm: Outcome on spasm and complications. A review. Neurochirurgie. 2018;64(2):106-116. doi: 10.1016/j. neuchi.2018.01.001. 